The Rangeland Journal, 2013, 35, 393-401

http://dx.doi.org/10.1071/RJ13011

\title{
Can competition with pasture be used to manipulate bellyache bush (Jatropha gossypiifolia L.) population biology?
}

\author{
Faiz F. Bebawi ${ }^{\mathrm{A}, \mathrm{C}}$, Shane D. Campbell ${ }^{\mathrm{A}}$ and Robert J. Mayer ${ }^{\mathrm{B}}$ \\ ABiosecurity Queensland, Department of Agriculture, Fisheries and Forestry, Tropical Weeds Research Centre, \\ PO Box 187, Charters Towers, Qld 4820, Australia. \\ ${ }^{B}$ Agri-Science Queensland, Department of Agriculture, Fisheries and Forestry, PO Box 1085, Townsville, \\ Qld 4810, Australia. \\ ${ }^{\mathrm{C} C}$ Corresponding author. Emails: Bebawif@gmail.com; Faiz.Bebawi@daff.qld.gov.au
}

\begin{abstract}
Bellyache bush (Jatropha gossypiifolia L.) is an invasive weed that poses economic and environmental problems in northern Australia. Competition between pasture and bellyache bush was examined in North Queensland using combinations of five pasture treatments (uncut (control); cut as low, medium, and high pasture; and no pasture) and four bellyache bush densities $\left(0,2,6\right.$ and 12 plants $\mathrm{m}^{-2}$ ) in a buffel grass (Cenchrus ciliaris L.) dominated pasture. The pasture treatments were applied approximately once per year but no treatments were applied directly to the bellyache bush plants. Measurements of bellyache bush flowering, seed formation, and mortality were undertaken over a 9-year period, along with monitoring the pasture basal cover and plant species diversity. Maximum flowering rates of bellyache bush occurred after 9 years $(97 \%)$ in plots containing no pasture, with the lowest rates of $9 \%$ in uncut control plots. Earliest flowering (322 days after planting) and seed formation (411 days) also occurred in plots with no pasture compared with all other pasture treatments (range 1314-1393 days for seed formation to occur). No seeds were produced in uncut plots. At the end of 9 years, mortality rates of bellyache bush plants initially planted averaged $\geq 73 \%$ for treatments with some pasture compared with 55\% under the no-pasture treatment. The percentage of herbaceous plant basal cover in uncut plots was increased 5-fold after 9 years, much greater than the average $2 \%$ increase recorded across the low, medium, and high pasture treatments. The number of herbaceous species in uncut plots remained largely unchanged, whereas there was an average reduction of $46 \%$ in the cut pasture treatments. Buffel grass remained the species with the greatest basal cover across all cut pasture treatments, followed by sabi grass (Urochloa mosambicensis (Hack.) Dandy) and then red Natal grass (Melinis repens (Willd.) Ziska). These results suggest that grazing strategies that maintain a healthy and competitive pasture layer may contribute to reducing the rate of spread of bellyache bush and complement traditional control techniques such as the use of herbicides.
\end{abstract}

Additional keywords: buffel grass, mortality, phenology, rangelands.

Received 6 February 2013, accepted 2 August 2013, published online 16 September 2013

\section{Introduction}

Bellyache bush (Jatropha gossypiifolia L.) was included among the 12 additional Weeds of National Significance in Australia in 2012 (Australian Weeds Committee 2012). It has the potential to form monocultures and thus reduce or eliminate pasture species from beneath its canopy. It can poison cattle, horses, and goats, especially in times of drought when other green fodder is not available (Csurhes 1999). It is a major pest of pastures in northern Australia (Parsons and Cuthbertson 2001; Bebawi et al. 2007, 2009). Thick stands inhibit the movement of stock and reduce the productivity of grazing areas, as it is woody and generally unpalatable to stock. In addition, its shallow root system has the potential to consume resources (water and nutrients) otherwise utilised by both introduced and native pasture species (Bebawi et al. 2007, 2009). Particularly in Queensland, it invades national parks as well as grazing land and so increases the cost of management of these lands (Tothill et al. 1982; Csurhes 1999; Parsons and Cuthbertson 2001). Bellyache bush is an important pest throughout the rangelands of northern Australia, extending across Queensland, the Northern Territory, and Western Australia (Bebawi et al. 2009).

Several control methods, including burning, slashing, stickraking, and foliar spraying, have been used to manage bellyache bush (Bebawi and Campbell 2002 a, $2002 b$; Vitelli and Madigan 2004; Bebawi et al. 2007, 2009, 2011; Randall et al. 2009). Treatment combinations over 4 years using foliar spraying either 
alone or as a follow-up with slashing, stick-raking, or burning have proven most successful for control of medium to large infestations of bellyache bush (Bebawi et al. 2011). Foliar spraying once each year for 4 years proved the most cost-effective control strategy, although it was acknowledged that more frequent treatment intervals might be required under highly favourable growth conditions, such as during above-average wet seasons (Bebawi et al. 2011). Other management strategies such as manipulating competing herbaceous species have not been investigated.

Pastures are the 'engines' that drive animal production systems and are the major source of nutrients for cattle in the rangelands of northern Australia (McIvor 2002). There are many reports of weeds increasing under heavy grazing (Tropical Forages 2011), e.g. honey mesquite (Prosopis glandulosa var. glandulosa) in the south-western United States (Buffington and Herbel 1965; Fisher 1977; Brown and Archer 1999). In addition, anecdotal evidence suggests that overgrazing generally renders pastures more susceptible to bellyache bush invasion, but it is important to note that pasture species may differ in their ability to resist weed invasion. For example, Thrasher et al. (1963) reported that forage crops using white clover (Trifolium repens L.) were more effective at suppressing weed populations of Canada thistle (Cirsium arvense (L.) Scop.) than of birdsfoot trefoil (Lotus corniculatus L.). In Australia, selected pasture plants including purple pigeon grass (Setaria incrassata (Hochst.) Hack), buffel grass (Cenchrus ciliaris L.), butterfly pea (Clitoria ternatea L.), kangaroo grass (Themeda triandra Forssk.), and Bull Mitchell grass (Astrebla squarrosa C.E. Hubb.) were able to displace parthenium weed (Parthenium hysterophorus L.) under field conditions, whereas Indian couch (Bothriochloa pertusa (L.) A.Camus) and Seca stylo (Stylosanthes scabra Vogel) were not able to do this (O'Donnell and Adkins 2005; Adkins et al. 2010; Khan et al. 2010). It is possible, therefore, that grazing management may be useful in suppressing the reproduction of bellyache bush, but some understanding of the dynamics between pasture grasses and bellyache bush in northern Australia is essential before such grazing strategies can be designed.

The objective of the present study was to examine the interaction between the basal cover, height, and diversity of associated pasture and the phenology and survival of bellyache bush. The hypothesis tested was that the basal cover and height of the associated pasture would affect the mortality, flowering, seed production, and general reproductive biology of bellyache bush in an ungrazed pasture over a period of 9 years.

\section{Materials and methods}

Site description

The experimental site $\left(20^{\circ} 01^{\prime} \mathrm{S}, 146^{\circ} 10^{\prime} \mathrm{E}\right.$; elevation $299 \mathrm{~m}$ asl) was on a grazing property $13 \mathrm{~km}$ north-west of Charters Towers, North Queensland. The site was open woodland with scattered trees and shrubs of native vegetation including narrow-leaved red ironbark (Eucalyptus crebra F.Muell.), carbeen (Corymbia tessellaris (F.Muell.) K.D. Hill and L.A.S. Johnson), whitewood (Atalaya hemiglauca (F.Muell) F.Muell ex Benth), false sandalwood (Eremophila mitchellii Benth.), red bauhinia [Lysiphyllum cunninghamii (Benth.) de Wit], white flowered bauhinia [L. hookeri (F.Muell.) Pedley], and yellowwood (Terminalia oblongata F.Muell.). Buffel grass dominated the pasture species. The three most common pasture species, buffel grass, Indian couch, and sabi grass (Urochloa mosambicensis (Hack.) Dandy), were in $100 \%, 38 \%$, and $28 \%$ of the plots, respectively. Pasture species identified on the site are listed in Table 1 and were all grasses. The area was fenced on 2 July 2002 to exclude grazing by native and domestic animals for the duration of the study.

Long-term mean annual rainfall for Charters Towers is $658 \mathrm{~mm}$, with $76 \%$ of this occurring during summer (DecemberFebruary) (BOM 2012). Rainfall at the field site was measured by an automatic weather station (Campbell Scientific, Logan, UT, USA). Rainfall was recorded for the duration of the trial (2002-11). Between 2002 and 2006, annual rainfall was consistently less than the long-term average for the area by $26 \%$, whereas between 2007 and 2011, it was consistently greater than the long-term average by $85 \%$. From 2002 to 2011, the site received 423, 512, 347, 565, 588, 1259, 1365, 1105, 1323 and $1037 \mathrm{~mm}^{\text {rain }}$ year $^{-1}$, respectively.

\section{Experimental design}

A two-factor experiment was established, comprising four densities of bellyache bush and five cutting regimes implemented to manipulate pasture biomass. The experiment used an 'additive' treatment design in 10 randomised blocks. The 'additive' design consisted of introducing bellyache bush plants into the pasture plots without destroying any of the existing pasture plants (except for a no-pasture treatment where all grass was removed) at a range of densities: control (nil plants), low $\left(2\right.$ plants $\left.\mathrm{m}^{-2}\right)$, medium $\left(6\right.$ plants $\left.\mathrm{m}^{-2}\right)$, and high $\left(12\right.$ plants $\left.\mathrm{m}^{-2}\right)$. The pasture treatments were: no pasture (pasture completely removed by cutting at ground level and placing weed matting on the soil surface to stop reshooting); low pasture (pasture cut at $10 \mathrm{~cm}$ height); medium pasture (pasture cut at $20 \mathrm{~cm}$ height); high pasture (pasture cut at $40 \mathrm{~cm}$ height); and uncut (control). The

Table 1. List of pasture species at the experimental site

\begin{tabular}{llll}
\hline Common name & Scientific name & Common name & Scientific name \\
\hline Buffel grass & Cenchrus ciliaris L. & Desert blue grass & Bothriochloa ewartiana (Domin) C.E. Hubb. \\
Golden beard grass & Chrysopogon fallax S.T. Blake & Sabi grass & Urochloa mosambicensis (Hack.) Dandy \\
Red Natal grass & Melinis repens (Willd.) Ziska & Black speargrass & Heteropogon contortus (L.) Beauv. Ex Roem. and Schult. \\
Dark wiregrass & Aristida calycina R.Br. & Green chloris & Chloris virgata Sw. \\
Indian couch & Bothriochloa pertusa (L.) A. Camus & Native couch & Brachyachne convergens (F.Muell.) Stapf. \\
Native millet & Panicum decompositum R.Br. & Brigalow grass & Paspalidium caespitosum C.E. Hubb. \\
\hline
\end{tabular}


experimental unit area was a permanent quadrat $\left(1 \mathrm{~m}^{2}\right)$ separated from adjacent permanent quadrats within the site by $1-\mathrm{m}$ paths kept free of pasture shoot systems through regular mowing $\sim 10 \mathrm{~cm}$ above ground.

\section{Treatment application}

Prior to the commencement of the trial, bellyache bush plants were raised (July-September 2002) at the Tropical Weeds Research Centre (Charters Towers) from seed sown in plastic pots filled with soil obtained from the vicinity of the experimental site. These plants were then transplanted into quadrats at the site in spring (September 2002) at the designated densities, and were $\sim 20 \mathrm{~cm}$ high at the time. Care was taken not to destroy any existing pasture grasses when these seedlings were planted out.

Pasture treatments were initiated on 14 February 2003 following completion of pasture composition assessments (Table 1) during the previous 2 days. Hand-held mechanical clippers (RYOBI Model OGS1820, Techtronic Industries Co. Ltd, Hong Kong) were used to cut pasture in the quadrats at the predetermined heights, with all cut material removed. The planted bellyache bushes were not disturbed. Frequency of cutting depended on the season and on how rapidly the pasture was growing at that time, with cuts normally performed once per year after the wet season because the pasture was dormant during the dry season. There was more than one cut in 2008 and 2010, which were the wettest years. The experiment was concluded on 18 May 2011, which as 9 years after transplanting the bellyache bush.

\section{Plant measurements}

Bellyache bush plants in each quadrat were monitored regularly for mortality and any flowering or seed formation. Plants were considered dead if they were shrivelled from top to bottom and the basal bark was cracked and split open. Once a whole plant dried up it was removed from the plot. Plant and seedling removal (see below) from the plots did not seriously disturb the topsoil because bellyache bush has a shallow root system (Parsons and Cuthbertson 2001; Bebawi et al. 2007, 2009). Times to flowering and seed formation were recorded when the first flower or seed was observed on an individual plant within the plots.

Pasture basal cover and diversity were also assessed for the 20 treatment combinations. A modified version of a 'cover pin frame' was used to measure basal cover of species making up the pasture composition, in which pins are held in a frame and moved up and down through a series of holes in two parallel bars, one fixed vertically above the other (Moore and Chapman 1986). The system used in this study was locally manufactured at the Tropical Weeds Research Centre. This sampling system was used in the first year when the bellyache bush plants were $<80 \mathrm{~cm}$ high. It was also used in the final year after all bellyache bush plants were removed to facilitate movement of the horizontal pin-holding bar. Moving the mobile pin holder 10 times across the plant quadrat allowed for 100 sample points $(10 \mathrm{~cm}$ by $10 \mathrm{~cm})$ per square metre for each plant quadrat. Whenever a pin made contact at ground level with the base of a tussock of a pasture grass, the species was recorded. Hence, the number of different species as well as the percentage basal cover of each was recorded for each quadrat. The number of different pasture species recorded in each plot was used as a measure of species diversity.

Any seedlings of bellyache bush germinating from seeds originating from adjacent infestations of bellyache bush, or which were naturally dispersed by maturing plants on-site, were regularly removed from the trial site (each time pastures were cut) to maintain the integrity of the original bellyache bush density treatments. Only seedlings recruited between 2010 and 2011 were counted to obtain an estimate of their population density.

\section{Statistical analysis}

Data collected from the plots were analysed as a two-factor factorial in 10 randomised blocks (GENSTAT 8.1; VSN International Ltd, Hemel Hempstead, UK). Fisher's protected least significant difference testing was used to determine differences between treatments.

\section{Results}

Table 2 presents $F$ - and $P$-values derived from analysis of variance to quantify significant differences and interactions associated with imposed pasture cutting and bellyache bush density treatments. Although significant differences were recorded between pasture treatments for several of the measurements, the low, medium, and high pasture treatments recorded similar responses. Consequently, these data have been pooled and pasture treatments are presented in the following sections as uncut pasture, cut pasture, or no pasture. The percentages of bellyache bush plants that flowered or seeded did not differ between the different densities, and so the data have been pooled for presentation (Fig. 1a, $b$ ).

\section{Percentages of bellyache bush flowering and seed formation}

There were significant differences $(P<0.001)$ in flowering percentages among pasture treatments across all years (Table 2 , Fig. 1a). At the end of 9 years, $97 \%$ of plants in the no-pasture plots had flowered compared with just $9 \%$ in uncut plots. Flowering levels in the cut pasture plots averaged $21 \%$ after 9 years. Moreover, flowering levels were not affected by bellyache bush densities $(P>0.05)$, and the above values are averages across the three non-zero density levels. By contrast, a highly significant interaction $(P<0.001)$ occurred between pasture treatments and the density of bellyache bush in relation to the percentage of bellyache bush plants that produced seed (Table 2, Fig. 1b). Bellyache bush plants in the uncut plots did not produce any seed by the end of the 9 years, whereas $11 \%$ of those in cut pastures had produced seed, irrespective of the density of bellyache bush. The no-pasture plots recorded the greatest percentage of bellyache bush plants that produced seed (94\%), although the density of bellyache bush had a significant effect. Seed formation levels were reduced by $5 \%$ at high density of bellyache bush compared with low and medium densities. 
Table 2. A summary of statistical values associated with pasture treatments (pooled), bellyache bush density treatments, and the interaction between pasture and bellyache bush treatments $F$, Observed variance; $P$, tabulated probability

\begin{tabular}{lcccc}
\hline Variable & Statistic & Pasture (P) & $\begin{array}{c}\text { Treatment } \\
\text { Bellyache bush (BAB) }\end{array}$ & P $\times$ BAB \\
\hline Flower incidence & $F$ & 323.5 & 2.16 & 2.29 \\
& $P$ & $<0.001$ & 0.120 & 0.063 \\
Seed formation incidence & $F$ & 718.4 & 3.38 & 7.83 \\
& $P$ & $<0.001$ & 0.037 & $<0.001$ \\
Days to flower & $F$ & 502.2 & 2.1 & 5.4 \\
& $P$ & $<0.001$ & 0.131 & $<0.001$ \\
Days to form seed & $F$ & 2018.1 & 5.9 & 13.8 \\
& $P$ & $<0.001$ & 0.004 & $<0.001$ \\
Mortality & $F$ & 49.0 & 0.72 & 1.89 \\
Pasture basal cover & $P$ & $<0.001$ & 0.49 & 0.12 \\
Pasture species diversity & $F$ & 1160.8 & 1.45 & 1.06 \\
& $P$ & $<0.001$ & 0.23 & 0.37 \\
& $F$ & 4.97 & 0.64 & 0.45 \\
& $P$ & 0.03 & 0.59 & 0.72 \\
\hline
\end{tabular}
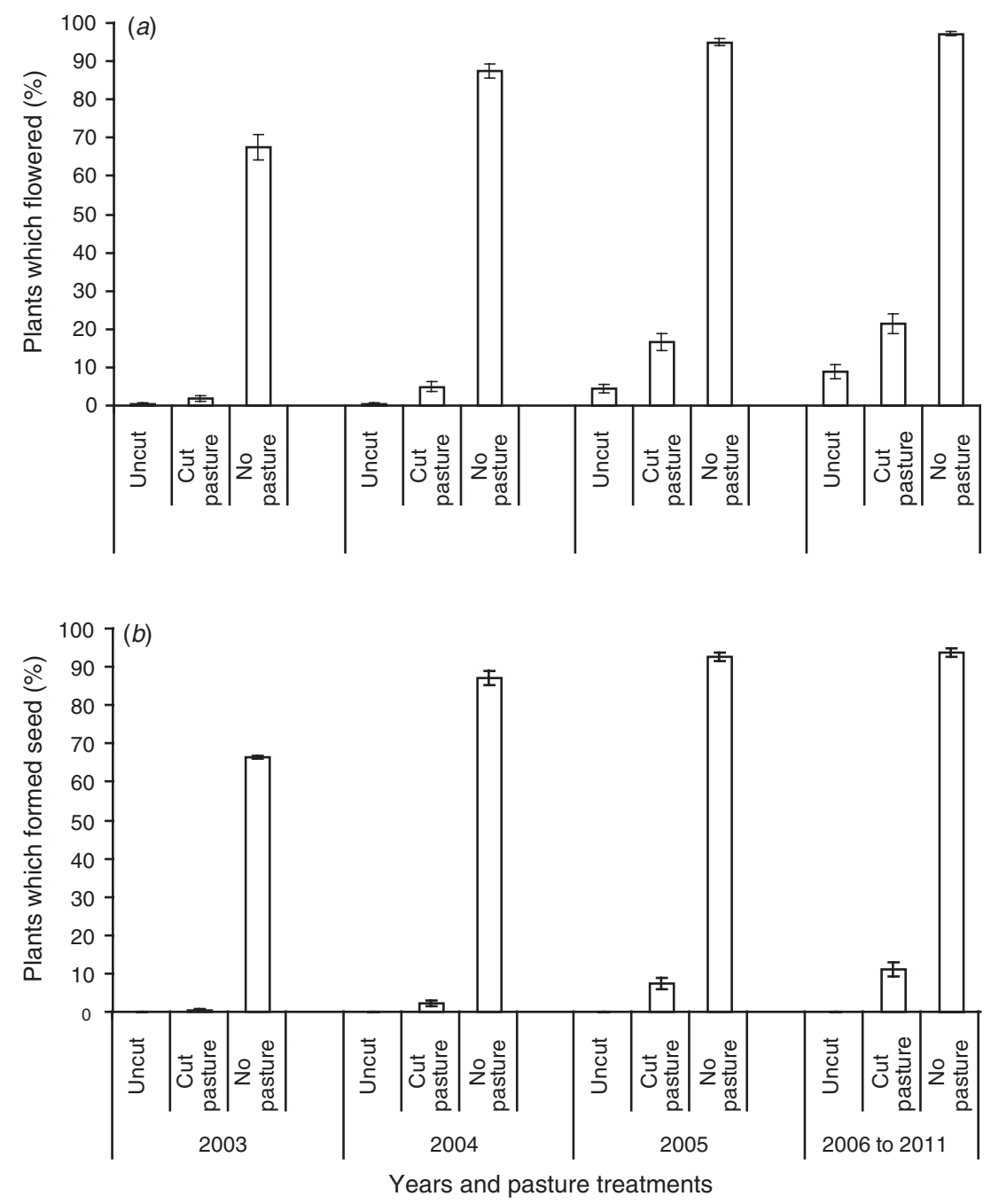

Fig. 1. Levels of ( $a$ ) flowering and $(b)$ seed production of bellyache bush averaged over three densities and exposed to different pasture treatments. Capped lines indicate standard errors of the means. 


\section{Number of days to flowering and seed formation of bellyache bush}

Significant interactions $(P<0.001)$ between pasture treatments and the density of bellyache bush occurred for both the number of days to flowering and the number of days to seed formation (Table 2, Fig. 2). Irrespective of density, bellyache bush plants took, on average, 1366 and 1295 days to flower in uncut and cut pasture treatments, respectively. Flowering of bellyache bush was much faster (322 days) where there was no pasture, but was significantly influenced by the density of bellyache bush. Under no-pasture conditions, the time to flowering at low, medium, and high densities of bellyache bush averaged 167, 296, and 504 days, respectively.

Bellyache bush plants did not produce seed in uncut plots during the trial; those in cut-pasture treatments took, on average, 1356 days to produce seeds, irrespective of the density of bellyache bush. As with time to flowering, seed formation was quickest when there was no pasture ( $<632$ days), particularly at the lowest density of bellyache bush (233 days).

\section{Bellyache bush mortality}

Mortality of bellyache bush was significantly $(P<0.001)$ affected by pasture treatments, but not by density of bellyache bush $(P>0.05)$ (Table 2). Progressive mortality rates of bellyache bush exposed to pasture treatments (averaged over all three densities) are shown in Fig. 3. For all pasture treatments except no pasture, mortality followed generally sigmoidal responses. In the no-pasture treatment, mortality remained low until a sharp increase in years 8 (2010) and 9 (2011). After 9 years, highest mortality of $78 \%$ occurred with cut pasture. This was closely followed by uncut pasture, which averaged $73 \%$, much higher than the $55 \%$ recorded across the no-pasture plots. Bellyache bush seedling recruitment density in 2010-11 averaged 37 seedlings $\mathrm{m}^{-2}$.

\section{Pasture cover}

Percentage basal cover of pasture plants was significantly $(P<0.001)$ affected by pasture treatments, but not density of bellyache bush $(P>0.05)$ (Table 2). In the uncut-pasture

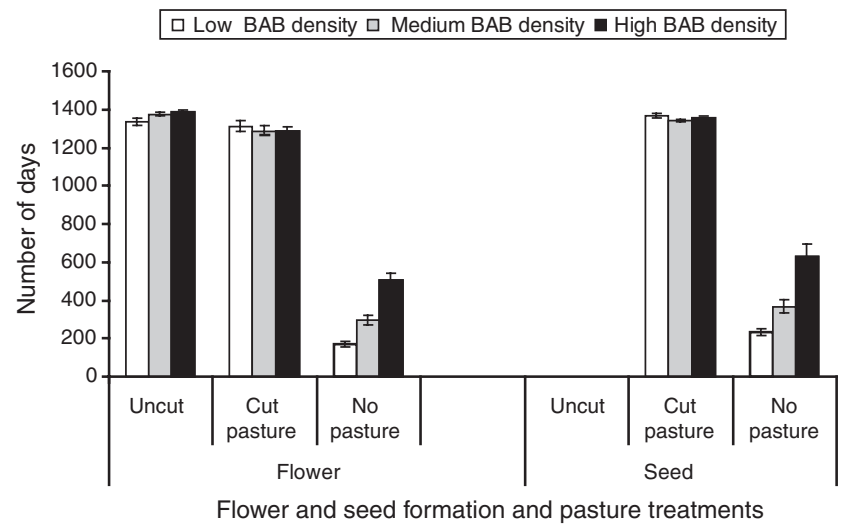

Fig. 2. Number of days to flowering and seed formation of bellyache bush at three densities exposed to different pasture treatments. Capped lines indicate the standard errors of the means. treatment, pasture basal cover increased from $13 \%$ to $71 \%$ over 9 years. By contrast, pasture basal cover increased by an average of $2 \%$ in the cut-pasture treatments (Fig. 4).

\section{Pasture species and diversity}

Pasture species diversity was significantly $(P<0.001)$ affected by pasture treatments, but not by density of bellyache bush $(P>0.05)$ (Table 2). Across all densities of bellyache bush, pasture species diversity changed little between the initial and final years in plots exposed to uncut conditions (Fig. 5), whereas the plots exposed to cut-pasture treatments experienced diversity reductions of $46 \%$. Of the 10 pasture species recorded at the start of the trial, four (buffel grass, red Natal grass, golden beard grass, and sabi grass) still occurred in the cut-pasture plots after 9 years (Fig. $6 a, b$ ). Of these, sabi grass was the only one that had a similar abundance, but buffel grass remained the dominant species. Indian couch, desert bluegrass, black spear grass, green chloris, and dark wiregrass were not found in any plots at the end of the trial, whereas native millet was only found in the uncut plots (Fig. 6b).

\section{Discussion}

This study has demonstrated the benefits of maintaining pasture to provide interspecific competition with bellyache bush.

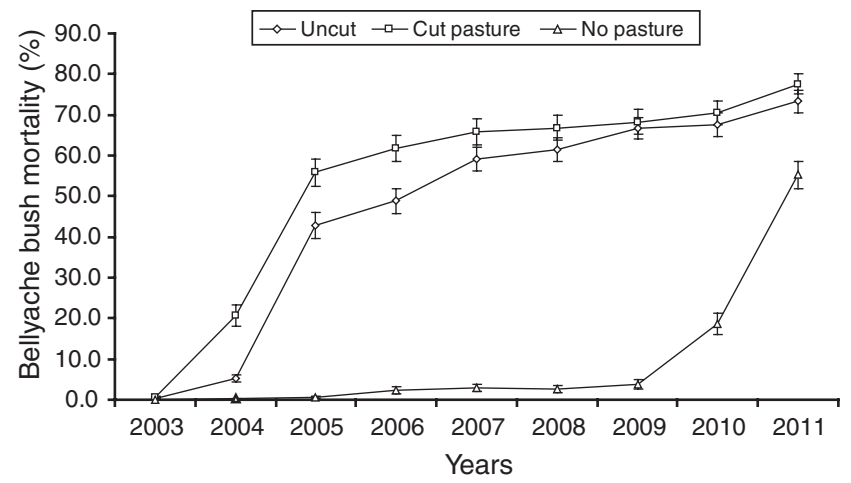

Fig. 3. Mortality of bellyache bush averaged over three densities as affected by pasture treatments over 9 years. Capped lines indicate the standard errors of the means.

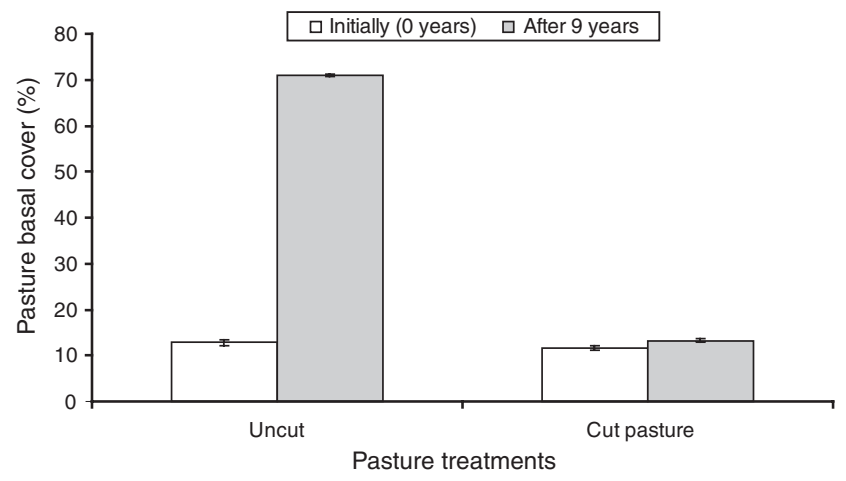

Fig. 4. Basal pasture cover (\%) initially (0) and at the end of 9 years under two pasture treatments averaged over all non-zero bellyache bush densities. Capped lines indicate the standard errors of the means. 


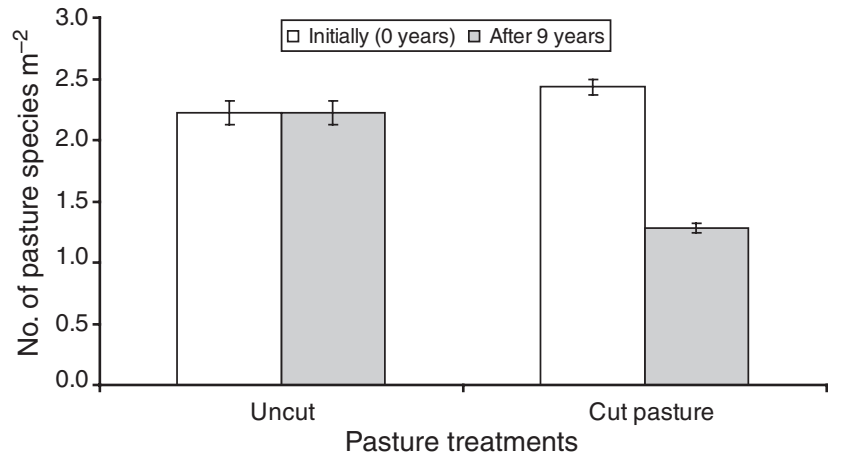

Fig. 5. Changes in diversity of pasture species over 9 years under two pasture treatments averaged over all bellyache bush densities. Capped lines indicate the standard errors of the means.

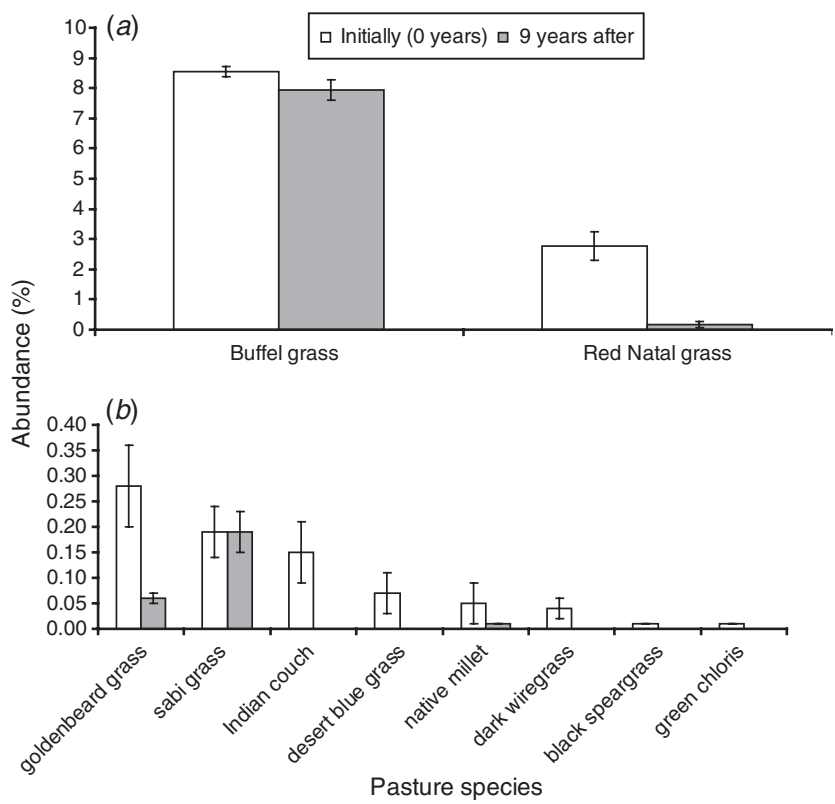

Fig. 6. Basal cover changes of (a) buffel grass and red Natal grass and (b) other grass species over 9 years averaged over uncut and cut pasture treatments and all bellyache bush densities. Capped lines indicate the standard errors of the means.

Compared with plots devoid of pasture, mortality of bellyache bush increased and reproduction declined when pasture was present. In the absence of pasture, intraspecific competition occurred, with reproduction reduced at the highest densities of bellyache bush plants. The study also showed that a buffel grass dominated pasture among bellyache bush was associated with an increase in pasture cover and a reduction in pasture species diversity in addition to greater mortality of bellyache bush.

\section{Levels of flowering and seed formation and time to flower and set-seed}

The incidence and rate of flowering and seed formation in bellyache bush was lowest in all treatments that contained pasture, particularly the uncut treatment where the pasture layer was not disturbed. Similar findings were reported in an earlier study (Bebawi et al. 2005), although the minimum number of days to flowering (average of 322 days in pasture-removed treatment) in the current study was considerably longer than reported under similar conditions where bare areas had been created following mechanical removal of bellyache bush (74 days). This difference may be attributed to differences in experimental conditions such as the dry years for the first 5 years of the experiment. The transplanting process may also have affected the time taken for bellyache bush to adjust to new environmental conditions.

In the absence of pasture (no-pasture treatment), intraspecific competition was prominent, with plants reaching reproductive maturity fastest at the lowest density of bellyache bush. This is consistent with the findings of a pot trial that compared flowering and pod production of bellyache bush grown at several densities (Bebawi et al. 2005).

These findings highlight the potential role of pasture-grazing management in modifying the dynamics of bellyache bush phenology and seed dispersal timing and, hence, population dynamics.

\section{Bellyache bush mortality}

Mortality of bellyache bush was highest in treatments that contained pasture, with little difference between cut and uncut treatments. However, whilst these findings demonstrate the importance of maintaining a pasture layer to compete with bellyache bush, and are consistent with findings for several other weeds and even some native woody species (Harrington et al. 1984; Scanlan et al. 1996; O'Donnell and Adkins 2005), they are not directly transferrable to actual grazing situations. The main difference is the frequency of grazing of individual plants, which could be several times annually under commercial grazing practices, compared with the single, end of wet season treatment implemented in the current study in most years, except in aboveaverage wet seasons (2008 and 2010). As such, even the most intense cut-pasture treatment in this study (i.e. low-pasture treatment where plants were cut off at $10 \mathrm{~cm}$ ) may be equivalent to only a light stocking rate in paddock grazed with domestic livestock.

The late introduction of bellyache bush plants into an already established pasture dominated by buffel grass most likely contributed to the high mortality of bellyache bush recorded in the pasture treatments; however, seedlings would encounter this situation in the early stages of invasion. Sagar (1959), working with Plantago lanceolata L. (a broad-leaved plant) and Lolium perenne L. (a grass), attributed the heavy mortality of either species to the time of sowing of the two species. Whichever species was sown late was greatly reduced in dry weight and it suffered much heavier mortality. Dissimilar growth habits, constitution, and structure may have resulted in unequal capture of light, whereby buffel grass may be greatly advantaged in terms of its leaf structure compared with bellyache bush. Additionally, the similarity of rooting habit, as both competing species have shallow root systems, may have resulted in greater competition for soil water and nutrient resources. Harper (1971) indicated that the demand for water and nutrients by a pair of species provides an opportunity for a resource in short supply to be shared unevenly. 
The reason for the large increase in mortality in the nopasture treatment over the last 2 years of the study is unclear but could be that bellyache bush was approaching its normal lifespan. Bellyache bush plants are perennial but do not appear to survive more than 10-15 years in the dry tropics of Queensland (F. Bebawi, unpubl. data). Also, during the last 5 years of the study some flooding of the site occurred, particularly in 2007, and could have caused some mortality of bellyache bush. These years also had high incidence of rainfall events leading to extended periods of wet soil conditions, a factor conducive to the spread of the soil-borne fungus Scytalidium dimidiatum (Penz.) Sutton and Dyko. This fungus is reported to cause canker and wilt of bellyache bush stems, particularly in flooded depressions (Tomley 2003; F. Bebawi, pers. obs.), and may have resulted in increased mortality at the site.

Importantly, the current study was undertaken in a pasture dominated by buffel grass on a relatively fertile soil, and findings may vary in native pastures where heavy grazing pressures have been shown to result in rapid pasture decline (Ash et al. 2011). Buffel grass evokes diverse opinions in Australia. Agriculturally, it is recognised as the most valuable introduced grass in arid and semi-arid tropical areas where it is widely sown for pasture and soil conservation (McIvor 2003). Environmentally, some regard buffel grass as one of Australia's worst weeds displacing native and endemic plants (Humphries et al. 1991; Griffin 1993; Low 1997; Jackson 2005). It is an aggressive coloniser of moist and dry habitats and is strongly competitive as an established plant (McIvor 2003). In lightly grazed pastures, the displacement of two pasture legumes, Stylosanthes hamata (L.) Taub. and $S$. scabra Vogel, in a relatively dry habitat at Hillgrove near Charters Towers was attributed to competition with buffel grass (Ash et al. 1997). This aggressive competition is consistent with the mortality of bellyache bush recorded in the current study under all treatments that contained pasture.

The removal of all new bellyache bush seedlings at the end of each dry season each year obviously played an important role in the competitive dynamics between pasture species and bellyache bush. The seedling recruitment population density of 37 seedlings $\mathrm{m}^{-2}$ reported in this study was in agreement with that in the unburnt controls ( 40 seedlings $\mathrm{m}^{-2}$ ) reported by Bebawi and Campbell (2002a). It is speculated that in heavily grazed situations where the competitive ability of pasture species is reduced, bellyache bush densities would increase over time through seedling recruitment, thereby compounding the level of competition on any remaining pasture species. Eventually, this would be expected to lead to the formation of monocultures of bellyache bush, which has been observed at numerous locations (Miller 1982; Randall et al. 2009). In the present study, buffel grass was able to rejuvenate itself continuously through tiller production, whereas the bellyache bush was going through the natural process of ageing as well as artificial loss of new recruits.

\section{Pasture diversity and pasture basal cover}

Averaged over all bellyache bush densities (including nil), species diversity was reduced by an average of $46 \%$ after 9 years where pasture-cutting regimes were practiced. This implies that species diversity may drastically change where continuous grazing occurs. Other workers (Hodgkinson 1995; McIvor and
Gardener 1995; Fynn and O'Connor 2000; Fuhlendorf et al. 2001; Ash et al. 2011) have suggested that changes in species composition may be accelerated when drought and continuous high grazing pressures occur concurrently. Those workers also indicated that continuous grazing was the major driver of changes in species composition. The decline in species diversity reported in the present study was also possibly due to the aggressiveness of the established buffel grass pastures (Smyth et al. 2009).

The dramatic increase in pasture basal cover in the uncut plots compared with very little increase in pasture basal cover $(2 \%)$ in all of the cut pasture treatments may also be a reflection of the aggressiveness of buffel grass. The present findings agree with other research showing that buffel grass displaces native and endemic pasture plants of Australia (Humphries et al. 1991; Griffin 1993; Low 1997; McIvor 1998; Fairfax and Fensham 2000; Franks 2002; Clarke et al. 2005; Grice 2006). Jackson (2005) also found that buffel grass-dominated sites had fewer herbaceous species than did non-buffel grass sites, and that species richness was negatively associated with increasing buffel grass biomass.

The reduction in pasture diversity observed in this study might also be attributed to allelopathy imposed by buffel grass and perhaps bellyache bush. Evidence of possible allelopathy by buffel grass was reported by Cheam (1984), Fulbright and Fulbright (1990), Csurhes (1999), and Parsons and Cuthbertson (2001). Failure of the invasive weed calotrope (Calotropis procera (Ait.) W.T.Ait.) to establish in areas with well-grown buffel grass was attributed to allelopathy (Cheam 1984). Up to $90 \%$ loss of stem dry weight occurred in calotrope seedlings grown in soil containing buffel grass roots. Mono-specific stands of bellyache bush have been reported to inhibit establishment of native plants (Csurhes 1999; F. Bebawi, pers. obs.).

\section{Possible implications for grazing management}

Although this experiment did not directly compare the effect of different densities of pasture on bellyache bush survival and growth, and the cutting regimes were not reflective of stocking rates in grazed pastures, it did demonstrate the value of maintaining pasture to provide interspecific competition with bellyache bush. Recently, significant research has focussed on development of grazing strategies to improve the long-term sustainability of grazing enterprises in rangeland environments. For instance, work in semiarid New South Wales has shown that intermittent grazing can inhibit shrub re-invasion and favour the establishment of native grasses following woody plant clearing (Alemseged et al. 2011). Other studies have demonstrated that implementation of lighter stocking rates than those traditionally implemented can increase the composition of desirable perennial pasture species, thereby enhancing the resilience of pastures while maintaining productivity (e.g. Ash et al. 2011). It is envisaged that such grazing strategies would also help minimise the spread of weeds such as bellyache bush and complement traditional control techniques such as the use of herbicides. In a large field trial, annual applications of a selective herbicide that removed bellyache bush but not the pasture layer proved the most effective strategy (Bebawi et al. 2011). Other techniques (such as the use of machinery) were equally effective 
at killing bellyache bush, but they disturbed the pasture layer, resulting in much greater seedling recruitment. The selective foliar herbicide treatment maintained pasture, which was then able to actively compete with bellyache bush and suppress its growth and development (Bebawi et al. 2011).

\section{Conclusion}

The results from this pasture cutting experiment indicate that grazing practices should be considered when developing management strategies for bellyache bush in pastoral areas, particularly those dominated by buffel grass. Survival, phenology, and reproduction rate of bellyache bush were all reduced under the pasture regimes applied. Whether similar findings would occur in pastures dominated by native grass species such as black speargrass, desert blue grass, and golden beard grass is unknown but plausible, and warrants investigation. Where the pasture is dominated by buffel grass, pasture diversity is reduced. This loss of diversity is possibly inevitable once buffel grass is established.

\section{Acknowledgements}

Thanks are extended to The Queensland Department of Agriculture, Fisheries and Forestry and the CRC for Australian Weed Management for providing financial support. Special thanks are extended to Ralph Woodard and family for allowing the use of their property for research purposes. We also thank Dr Joe Scanlan, Mrs Barbara Madigan, Dr Wayne Vogler, and Dr Peter O'Reagain for reviewing the manuscript. The technical assistance of C. Crowley, K. Thorp, R. Stevenson, K. Risdale, and C. Andersen is also acknowledged.

\section{References}

Adkins, S. W., O'Donnell, C., Khan, N., Nguyen, T., Shabbir, A., Dhileepan, K., George, D., and Navie, S. (2010). Parthenium weed (Parthenium hysterophorus L.) research in Australia: new management possibilities. In: 'Proceedings 17th Australasian Weeds Conference'. (Ed. S. M. Zydenbos.) pp. 120-123. (New Zealand Plant Protection Society: Christchurch, New Zealand.)

Alemseged, Y., Hacker, R. B., Smith, W. J., and Melville, G. J. (2011). Temporary cropping in semi-arid shrublands increases native perennial grasses. The Rangeland Journal 33, 67-78. doi:10.1071/RJ10022

Ash, A. J., McIvor, J. G., Mott, J. J., and Andrew, M. H. (1997). Building grass castles: integrating ecology and management of Australia's tropical tallgrass rangelands. The Rangeland Journal 19, 123-144. doi:10.1071/ RJ9970123

Ash, A. J., Corfield, J. P., McIvor, J. G., and Ksiksi, T. S. (2011). Grazing management in tropical savannas: utilization and rest strategies to manipulate rangeland condition. Rangeland Ecology and Management 64, 223-239. doi:10.2111/REM-D-09-00111.1

Australian Weeds Committee (2012). 'Weeds of National Significance (2012).' (Department of Agriculture, Fisheries and Forestry: Canberra, ACT.)

Bebawi, F. F., and Campbell, S. D. (2002a). Impact of fire on bellyache bush (Jatropha gossypiifolia) plant mortality and seedling recruitment. Tropical Grasslands 36, 129-137.

Bebawi, F. F., and Campbell, S. D. (2002b). Effects of fire on germination and viability of bellyache bush (Jatropha gossypiifolia). Australian Journal of Experimental Agriculture 42, 1063-1069. doi:10.1071/ EA01125
Bebawi, F. F., Mayer, R. J., and Campbell, S. D. (2005). Flowering and capsule production of bellyache bush (Jatropha gossypiifolia L.) in northern Queensland. Plant Protection Quarterly 20, 129-132.

Bebawi, F. F., Vitelli, J. S., Campbell, S. D., Vogler, W. D., Lockett, C. J., Grace, B. S., Lukitsch, B., and Heard, T. A. (2007). The biology of Australian weeds 47. Jatropha gossypiifolia L. Plant Protection Quarterly 22, 42-58.

Bebawi, F. F., Vitelli, J. S., Campbell, S. D., Vogler, W. D., Lockett, C. J., Grace, B. S., Lukitsch, B., and Heard, T. A. (2009). Jatropha gossypiifolia L. In: 'The Biology of Australian Weeds, Vol. 3'. (Ed. F. D. Panetta.) pp. 102-127. (R. G. and F. J. Richardson: Melbourne, Vic.)

Bebawi, F. F., Vitelli, J. S., Campbell, S. D., and Mayer, R. J. (2011). Impact of control strategies on bellyache bush (Jatropha gossypiifolia L.) mortality, seedling recruitment, population dynamics, pasture yield and cost analysis. The Rangeland Journal 33, 277-286. doi:10.1071/ RJ10038

BOM (2012). Mean annual rainfall and maximum daily temperature ranges. Available at: www.bom.gov.au.

Brown, J. R., and Archer, S. (1999). Shrub invasion of grassland: recruitment is continuous and not regulated by herbaceous biomass or density. Ecology 80, 2385-2396. doi:10.1890/0012-9658(1999)080 [2385:SIOGRI]2.0.CO;2

Buffington, L. C., and Herbel, C. H. (1965). Vegetation changes on a semidesert grassland range. Ecological Monographs 35, 139-164. doi: $10.2307 / 1948415$

Cheam, A. H. (1984). Allelopathy in buffel grass (Cenchrus ciliaris L.) Part 1. Influence of buffel grass association on calotrope [Calotropis procera (Ait.) W.T.Ait.]. Australian Weeds 3, 133-136.

Clarke, P. J., Latz, P. K., and Albrecht, D. E. (2005). Long-term changes in semi-arid vegetation: Invasion of an exotic perennial grass has larger effects than rainfall variability. Journal of Vegetation Science 16, 237-248. doi:10.1111/j.1654-1103.2005.tb02361.x

Csurhes, S. M. (1999). 'Bellyache Bush (Jatropha gossypiifolia) in Queensland.' Pest Status Review Series - Land Protection. (Queensland Department of Natural Resources: Brisbane, Qld.)

Fairfax, R. J., and Fensham, R. J. (2000). The effect of exotic pasture development on floristic diversity in Central Queensland, Australia. Biological Conservation 94, 11-21. doi:10.1016/S0006-3207(99)00169-X

Fisher, C. E. (1977). Mesquite and modern man in south western North America. In: 'Mesquite: Its Biology in two Desert Scrub Ecosystems'. (Ed. B. B. Simpson.) pp. 177-188. (Dowden, Hutchinson \& Ross: Stroudsburg, PA.)

Franks, A. J. (2002). The ecological consequences of buffel grass Cenchrus ciliaris establishment within remnant vegetation of Queensland. Pacific Conservation Biology 8, 99-107.

Fuhlendorf, S. D., Briske, D. D., and Smeins, F. E. (2001). Herbaceous vegetation change in variable rangeland environments: the relative contribution of grazing and climatic variability. Applied Vegetation Science 4, 177-188. doi:10.1111/j.1654-109X.2001.tb00486.x

Fulbright, N., and Fulbright, T. E. (1990). Germination of 2 legumes in leachate from introduced grasses. Journal of Range Management 43, 466-467. doi: $10.2307 / 3899014$

Fynn, R. W. S., and O'Connor, T. G. (2000). Effect of stocking rate and rainfall on rangeland dynamics and cattle performance in a semi-arid savanna. South African Journal of Applied Ecology 37, 491-507.

Grice, A. C. (2006). The impact of invasive plant species on the biodiversity of Australian Rangelands. The Rangeland Journal 28, 27-35. doi:10.1071/ RJ06014

Griffin, G. F. (1993). The spread of buffel grass in inland Australia: Land use conflict. In: 'Weeds, Weed Control, Herbicides, Australasia. Proceedings of the 10th Australian Weeds Conference and the 14th Asian-Pacific Weed Science Society Conference'. (Ed. J. T. Swarbrick.) pp. 401-504. (The Weed Society of Queensland: Brisbane, Qld.) 
Harper, J. L. (1971). 'Population Biology of Plants.' (Academic Press: London.)

Harrington, G. N., Mills, D. M. D., Pressland, A., and Hodgkinson, K. C. (1984). Semi-arid woodlands. In: 'Management of Australia's Rangelands'. (Eds G. N. Harrington, A. D. Wilson and M. D. Young.) pp. 189-207. (CSIRO: East Melbourne, Vic.)

Hodgkinson, K. (1995). A model for perennial grass mortality under grazing. In: 'Rangelands in a Sustainable Biosphere. Proceedings of the Fifth International Rangeland Congress'. Denver, CO. (Ed. N. E. West.) pp. 240-241. (Society for Range Management: Denver, CO.)

Humphries, S. E., Groves, R. H., and Mitchell, D. S. (1991). Plant invasions: The incidence of environmental weeds in Australia. Kowari 2, 1-134.

Jackson, J. (2005). Is there a relationship between herbaceous species richness and buffel grass (Cenchrus ciliaris)? Austral Ecology 30, 505-517. doi:10.1111/j.1442-9993.2005.01465.x

Khan, N., O’ Donnell, C., Shabbir, A., and Adkins, S. W. (2010). Competitive displacement of parthenium weed with beneficial native and introduced pasture plants in central Queensland, Australia. In: 'New Frontiers in New Zealand: Together We Can Beat the Weeds. Proceedings 17th Australasian Weeds Conference'. (Ed. S. M. Zydenbos.) pp. 131-134. (New Zealand Plant Protection Society: Christchurch, New Zealand.)

Low, T. (1997). Tropical pasture plants as weeds. Tropical Grasslands 31, 337-343.

McIvor, J. G. (1998). Pasture management in semi-arid tropical woodlands: effects on species diversity. Australian Journal of Ecology 23, 349-364. doi:10.1111/j.1442-9993.1998.tb00740.x

McIvor, J. G. (2002). Pastures. In: 'Managing and Conserving Grassy Woodlands'. (Eds S. McIntyre, J. G. McIvor and K. M. Heard.) pp. 61-77. (CSIRO Publishing: Melbourne, Vic.)

McIvor, J. G. (2003). Competition affects survival and growth of buffel grass seedlings - is buffel grass a coloniser or an invader? Tropical Grasslands 37, 176-181.

McIvor, J. G., and Gardener, C. J. (1995). Pasture management in semiarid tropical woodlands: effects on herbage yields and botanical composition. Australian Journal of Experimental Agriculture 35, 705-715. doi:10.1071/EA9950705

Miller, I. L. (1982). 'Weeds in the Northern Territory: current situation and future directions.' (Northern Territory Department of Primary Production, Division of Agriculture and Stock: Darwin, NT.)
Moore, P. D., and Chapman, S. B. (1986). 'Methods in Plant Ecology.' (Blackwell Scientific Publications: London.)

O'Donnell, C., and Adkins, S. W. (2005). Management of parthenium weed through competitive displacement with beneficial plants. Weed Biology and Management 5, 77-79. doi:10.1111/j.1445-6664.2005. 00160.x

Parsons, W. T., and Cuthbertson, E. G. (2001). 'Noxious Weeds of Australia.' 2nd edn. (CSIRO, Publishing: Melbourne, Vic.)

Randall, A., Campbell, S., Vogler, W., Bebawi, F., and Madigan, B. (2009). 'Bellyache Bush (Jatropha gossypiifolia) Management Manual —Current Control Options and Management Case Studies from Across Australia.' (Queensland Primary Industries and Fisheries, Department of Employment, Economic Development and Innovation: Brisbane, Qld.)

Sagar, G. R. (1959). The biology of some sympatric species of grassland. PhD Thesis, University of Oxford, UK.

Scanlan, J. C., Pressland, A. J., and Myles, D. J. (1996). Grazing modifies woody and herbaceous components of North Queensland woodlands. The Rangeland Journal 18, 47-57. doi:10.1071/RJ9960047

Smyth, A., Friedel, M., and O'Malley, C. (2009). The influence of buffel grass (Cenchrus ciliaris) on biodiversity in an arid Australian landscape. The Rangeland Journal 31, 307-320. doi:10.1071/RJ08026

Thrasher, F. P., Cooper, C. S., and Hodgson, J. M. (1963). Competition of forage species with Canada thistle as affected by irrigation and nitrogen levels. Weeds 11, 136-138. doi:10.2307/4040707

Tomley, A. (2003). 'Bellyache Bush Jatropha gossypiifolia Infection by Scytalidium dimidiatum.' (Queensland Department of Natural Resources and Mines Office Memo: Brisbane, Qld.)

Tothill, J. C., Mott, J. J., and Gillard, P. (1982). Pasture weeds of the tropics and subtropics with special reference to Australia. In: 'Biology and Ecology of Weeds'. (Eds W. Holzner and N. Numata.) pp. 403-422. (Dr W. Junk Publishers: The Hague, the Netherlands.)

Tropical Forages (2011). Forage fact sheet-Brachiaria humidicola. Available at: www.tropicalforages.info.

Vitelli, J. S., and Madigan, B. A. (2004). Evaluation of a hand-held burner for the control of woody weeds by flaming. Australian Journal of Experimental Agriculture 44, 75-81. doi:10.1071/EA02096 